\title{
Separation Control of NACA0015 Airfoil using Plasma Actuators
}

Akira Aiura*, Kentaro Kobayashi, Jun Sakakibara

\author{
Department of Mechanical Engineering, Meiji University
}

Higashimita 1-1-1, Tamaku, Kawasaki, 214-8571, Japan

\section{ABSTRACT}

Separation control of NACA0015 airfoil using plasma actuators was investigated. Plasma actuators in spanwise array, which consists of 21 electrodes, were located at the leading edge of the airfoil to give temporal periodic disturbances with phase variations into its boundary layer. The cord length of the airfoil was $\mathrm{c}=100 \mathrm{~mm}$ and corresponding Reynolds number was fixed at $\mathrm{Re}=63,000$. Non-dimensional frequency of the disturbance was chosen at $F^{+}=0.5$ or 6 . The gap between adjacent electrode was set as $1 \mathrm{~mm}$, and phase difference of the temporal periodic disturbances between adjacent electrode was set at $\varphi=0$ or $\pi$. Velocity field was measured by conventional two-component PIV using a CCD camera (Imperx, B1922, 1920 x 1460pixels) and Nd-YAG laser (Quantel, Evergreen, 140mJ/pulse). Both large field of view (FOV) images capturing whole wing with surrounding flow and smaller FOV images focused on the separation bubble near leading edge were evaluated. Surface pressure was monitored by pressure transducers through pressure taps on the upper surface of airfoil. Lift and drag against the airfoil were measured using a twocomponent force balance.

Figure 1 shows a lift coefficient profile of airfoil against angles of attack $\alpha$. Before a stall angle found at $\alpha=11.25$ degrees, improvements of lift coefficients are confirmed in a range from $\alpha=9$ to 11 degrees under conditions of PA-on compared to that of PA-off. Besides, lift coefficients under the conditions of $F^{+}=6$ are greater than that of $F^{+}=0.5$. Among conditions of $F^{+}=6$, the $\varphi=\pi$ case provide the higher lift compared to the $\varphi=0$ case by approximately $1.4 \%$ at $\alpha=11$ degrees. Figure 2 shows a pressure profile on the surface of airfoil at $\alpha=11$ degrees. In addition to experimental values measured by the transducer shown by red markers, values estimated from large FOV's PIV data are shown by blue lines. Here, the pressure gradient directly computed from the measured velocity field based on mean momentum equation was spatially integrated from a point where the pressure was prescribed through a spatial marching procedure. Pressures near leading edge are clearly different between cases, while not much difference is found downstream. Maximum negative pressure values measured by the transducer is found under a PA-off case at $x=-0.07 \mathrm{c}$, which was the most upstream location of the pressure tap available to place. However, such a predominance of the PA-off case is not maintained towards leading edge, i.e. the negative pressure value under the $\varphi=\pi$ case exceeds others in a range from $x=-0.1 \mathrm{c}$ to $-0.2 \mathrm{c}$, as indicated by PIV based pressure. Figure 3 shows mean velocity distributions evaluated from the smaller FOV images. Separation bubbles are confirmed on conditions of PA-off and $F^{+}=6$. Comparing a condition of PA-off and conditions of $F^{+}=6$, the separation bubbles at $F^{+}=6$ cases are smaller than at a PA-off case. This might contribute to the increases of the lift coefficients, and it may be clarified through a pressure field estimated by PIV data based on the smaller 
FOV images. Figure 4 shows surfaces of constant vorticity magnitude extracted from a stack of phaseaveraged planer velocity field at $\alpha=11$ degrees. While quasi-spanwise-oriented roller vortices are formed in a $\varphi=0$ case, vortices arranged in a staggered manner are formed in a $\varphi=\pi$ case. Here, the layer of the vortices consists of series of lambda vortices, where the heads of each vortex are tilted away from the surface due to self-induction.

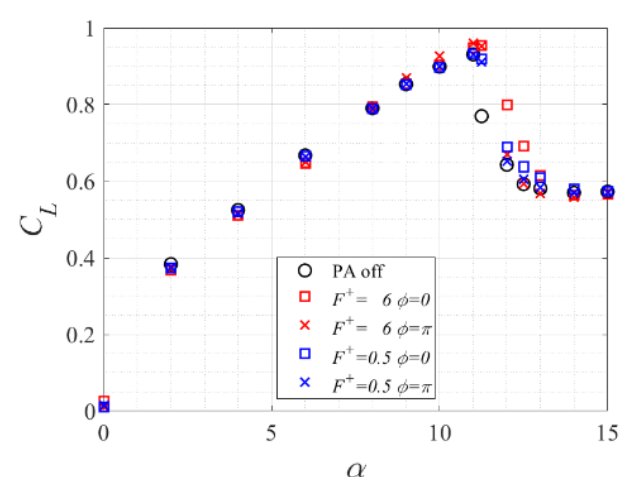

Fig. 1 A lift coefficient profile of airfoil against angles of attack.

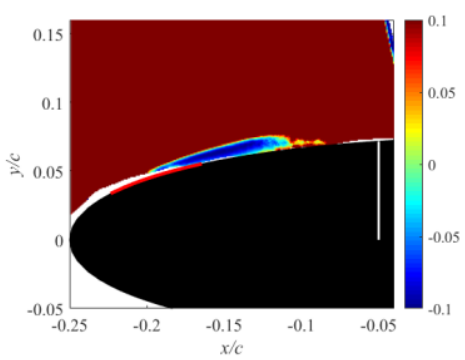

(a) PA off

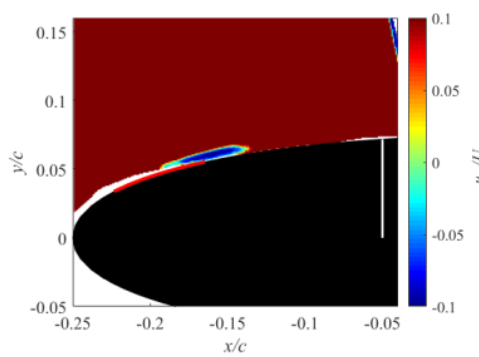

(b) $F^{+}=6, \varphi=0$

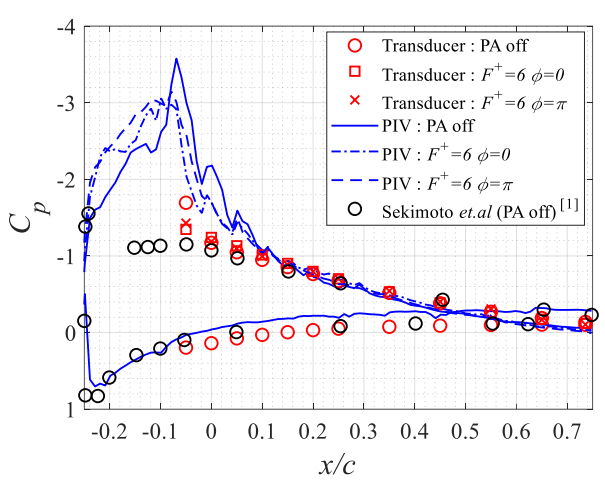

Fig. 2 A pressure profile on the surface of airfoil at $\alpha=11$ degrees.

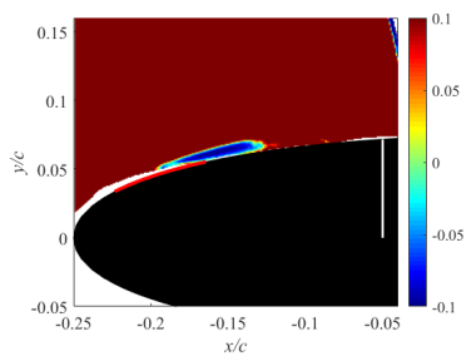

(c) $F^{+}=6, \varphi=\pi$

Fig. 3 Mean velocity distributions near leading edge at $\alpha=11$ degrees.

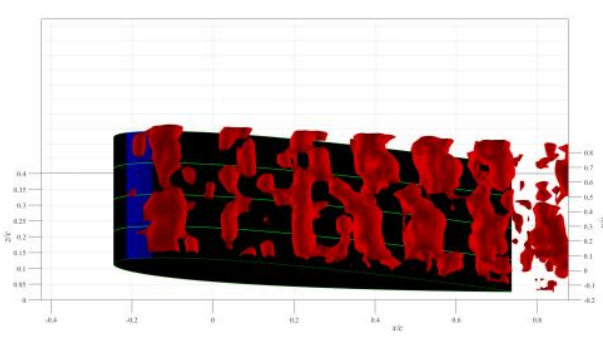

(a) $F^{+}=6, \varphi=0$

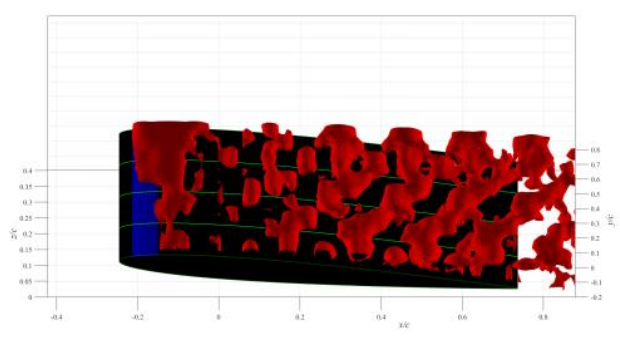

(b) $F^{+}=6, \varphi=\pi$

Fig. 4 Surface of constant vorticity magnitude $\left|Q c / U_{\infty}\right|=12$ at $\alpha=11$ degrees.

\section{REFERENCES}

[1] Sekimoto S., Nonomura T., Fujii K., 2017, Burst-Mode Frequency Effects of Dielectric Barrier Discharge Plasma Actuator for Separation Control, J. AIAA, 55, pp.1385-1392. 